17-700.R3 Coates edited UK English

\title{
Molecular analysis of PALB2 associated breast cancers
}

Jue Er Amanda Lee ${ }^{1}, \mathrm{Na} \mathrm{Li}^{1,2,3}$, Simone M. Rowley ${ }^{1}$, Dane Cheasley ${ }^{1,3}$, Magnus

Zethoven $^{4}$, Simone Mclnerny ${ }^{5}$, Kylie L. Gorringe ${ }^{3,6,7}$, Paul A. James ${ }^{3,5, *}$ and lan G.

Campbell ${ }^{1,3,7, *}$

${ }^{1}$ Cancer Genetics Laboratory, Peter MacCallum Cancer Centre, Melbourne, Victoria, Australia;

${ }^{2}$ Cancer Biology Medical Center, Tongji Hospital, Tongji Medical College, Huazhong University of Science and Technology, Wuhan, Hubei, China;

${ }^{3}$ Sir Peter MacCallum Department of Oncology, University of Melbourne, Melbourne, Victoria, Australia;

${ }^{4}$ Bioinformatics Consulting Core, Peter MacCallum Cancer Centre, Melbourne, Victoria, Australia;

${ }^{5}$ Parkville Familial Cancer Centre, Peter MacCallum Cancer Centre and Royal Melbourne Hospital, Melbourne, Victoria, Australia;

${ }^{6}$ Cancer Genomics Program, Peter MacCallum Cancer Centre, Melbourne, Victoria, Australia

${ }^{7}$ Department of Pathology, University of Melbourne, Melbourne, Victoria, Australia;

This is the author manuscript accepted for publication and has undergone full peer review but has not been through the copyediting, typesetting, pagination and proofreading process, which may lead to differences between this version and the Version of Record. Please cite this article as doi: $10.1002 /$ path.5055

This article is protected by copyright. All rights reserved. 
*These Authors contributed equally

Correspondence to: Ian Campbell, Cancer Genetics Laboratory, Research Division, Peter MacCallum Cancer Centre, 305 Grattan Street, Melbourne, Victoria, 3000, Australia. E-mail: ian.campbell@petermac.org.

\section{Conflict of interest statements}

No conflicts of interest were declared.

Running title: Molecular analysis of PALB2 associated breast cancers 


\section{Abstract}

PALB2 is established as the most clinically important moderate to high penetrance breast cancer predisposition gene after $B R C A 1$ and $B R C A 2$. Mutations in classical familial cancer predisposition genes are presumed to be recessive at the cellular level and therefore a second inactivating somatic mutation is required in the tumour tissue. However, from the limited data that exists, PALB2 may be an example of a cancer predisposition gene that does not conform to Knudsen's "two hit" paradigm. We conducted genome-wide copy number analysis and targeted sequencing of PALB2 and other breast cancer driver genes in 15 invasive breast cancers from individuals carrying pathogenic germline mutations in PALB2. The majority of cancers showed clear evidence of bi-allelic inactivation of PALB2 (10/15) either as loss of heterozygosity involving the wild-type allele (6 tumours) or somatic point mutations (4 tumours). All PALB2 null cancers had high homologous recombination deficiency (HRD) scores consistent with a homologous recombination repair deficiency. Interestingly, all but one of the PALB2 heterozygous cancers also had high HRD scores suggesting alternative mechanisms of PALB2 functional loss might be operating in these cancers. Our findings demonstrate that PALB2 does undergo bi-allelic inactivation in the majority of breast cancers from PALB2 germline mutation carriers. This feature has implications for the discovery of new moderate to high penetrance breast cancer predisposition genes as it supports using the existence of a "second hit" and mutation signatures as important search criteria. 


\section{Keywords}

PALB2; Loss of heterozygosity; tumour suppressor gene; homologous recombination deficiency

\section{Introduction}

PALB2 was first identified as a BRCA2-interacting protein and involved in homologous recombination-mediated repair of double-strand DNA breaks [1]. Bi-allelic mutations in PALB2 are responsible for Fanconi anaemia complementation group $\mathrm{N}[2,3]$ while monoallelic mutations are associated with increased breast cancer susceptibility [4]. PALB2 is now established as the most clinically important breast cancer predisposition gene after $B R C A 1$ and BRCA2 [5].

Mutations in classical familial cancer predisposition genes are presumed to be recessive at the cellular level and therefore require a second somatic inactivating mutation in the tumour according to the model first proposed by Knudson from his observations of familial retinoblastoma [6]. In the search for new moderate to high penetrance familial breast cancer genes, the absence of a "second hit" in a candidate gene has generally been considered sufficient to nullify its candidacy. However, since its discovery, PALB2 has been cited as an example of a predisposition gene that may not require bi-allelic inactivation in tumours [7-9]. Data from seven studies that have collectively analysed 20 breast tumours from germline carriers of PALB2 loss of function (LoF) mutations [7-13] provided mixed results but overall over $50 \%$ retained both the wild-type and mutant PALB2 alleles (Table 1) although for one study a second nonsense somatic mutation was 
detected [13]. Since only one of the seven studies sequenced the entire coding region of the PALB2 gene in the tumours, the presence of second somatic point mutations cannot be excluded and it is consequently premature to conclude that PALB2 does not undergo bi-allelic inactivation. Resolving this issue has important implications for the strategies that should be adopted to identify the genes responsible for the $\sim 50 \%$ of breast cancer families that are not explained by known predisposition genes.

In order to determine whether PALB2 requires bi-allelic inactivation and if this is associated with a characteristic genomic scar signature, we analysed DNA extracted from 15 breast cancer tumours from women with a PALB2 germline loss of function (LoF) mutation. 


\section{Materials and methods}

\section{Breast tumour cohort}

PALB2 LoF mutation carrier families were identified from the Variants in Practice (ViP) Study through the Victorian and Tasmanian Familial Cancer Clinics, Australia. The family and/or personal history of all the cases was assessed by a specialist Familial Cancer Clinic and determined to be sufficiently strong to be eligible for clinical genetic testing by local criteria. All cases tested negative for pathogenic mutations in $B R C A 1, B R C A 2$ and other hereditary breast and ovarian cancer genes as described previously [14]. Fifteen cases where an actionable germline LoF PALB2 was identified also had formalin fixed paraffin embedded (FFPE) breast cancer biopsies available. Carrier subject details and tumour characteristics obtained from pathology reports are listed in Table 2 and Supplementary Table S1. All participants provided informed consent for genetic analysis of their germline and tumour DNA. This study was approved by the Human Research Ethics Committees at each participating ViP study recruitment centre and the Peter MacCallum Cancer Centre (Approval \# 09/29).

\section{Tumour DNA extraction}

For each FFPE tumour, a representative haematoxylin and eosin (H\&E) stained section was prepared and used as a template to needle microdissect cancer cells from H\&E stained $10 \mu \mathrm{m}$ sections. DNA was extracted using the QIAamp DNA FFPE Tissue Kit (Qiagen, Valencia, CA, USA) and quantified using Qubit dsDNA high-sensitivity Assay kit (ThermoFisher Scientific, Australia Pty Ltd). 


\section{Targeted sequencing library preparation, enrichment and sequencing}

Targeted sequencing of tumour DNA was performed using an Agilent SureSelect XT Custom Panel which targeted 487 genes (total targeted region of $1.337 \mathrm{Mb}$ ) including BRCA1, BRCA2, PTEN, ATM, ATR, BRIP1 and RAD51C and the entire coding region and introns of PALB2 spanning coordinates chr16:23614482-23657678 (Homo sapiens, hg19, GRCh37, February 2009). Library preparation was performed from an input of $300 \mathrm{ng}$ of tumour DNA using the KAPA Hyper system (Agilent, Santa Clara, USA). Sequencing of target-enriched DNA libraries were performed using the Illumina Next Seq 500 generating 75 bp paired-end sequence reads.

\section{Variant calling, somatic mutation detection and genome-wide copy number analysis}

Paired-end sequence reads were aligned to the g1k v37 hg19 reference genome using BWA [15]. Optical duplicate reads were removed using Picard (http://broadinstitute.github.io/picard/), then local realignment around indels and base quality score recalibration were performed using the Genome Analysis Tool Kit (GATK) in accordance with their recommended best practice workflow [16]. SNP and indel variants were called using GATK UnifiedGenotyper, Platypus [17] and Varscan 2 [18]. Called variants were additionally annotated using the Ensembl Variant Effect Predictor [19]. Somatic mutations in the tumour sequencing data were identified by removing previously available germline variant data for hereditary breast and ovarian cancer panel genes, including PALB2 [14] and where this information was not available, by applying the following filters: canonical transcript; bidirectional read; quality $\geq 100$; variants identified by 
at least two variant callers; minor allele frequency (MAF) present at $\leq 0.0001$ in ExAc (Version 0.3.1, excluding TCGA data, released March 14 2016) [20], GnomAD (Version 2.0, released 27 February 2017) [20], EVS (Version ESP6500SI-V2-SSA137) [21] and 1000 Genomes [22] databases.

Off-target sequencing reads were used to generate genome-wide copy number (CN) data using copywriteR [23] utilising a normal lymphocyte DNA control (NA12878, Coriell Institute) run in the same sequencing batch for the normalisation baseline. Data was then imported to NEXUS Copy Number ${ }^{T M}$ (software v8.0 with build version 9169, BioDiscovery Inc), segmented using a FASST2 segmentation algorithm and visualized.

\section{Analysis of Loss-of-Heterozygosity (LOH) across chromosome 16}

In addition to copy number data, allelic information across the PALB2 locus on chromosome 16 was used to substantiate $\mathrm{LOH}$. High confidence germline heterozygous SNPs across chromosome 16 were identified from germline DNA data generated previously [14] using the following filters: canonical transcript; called by two or more callers; bidirectional read; quality $\geq 50$; present in one or more population databases ExAc, GnomAD, EVS and 1000 Genomes. The alternate allele frequency of these SNPs in the tumour tissue were then used to infer allelic status.

\section{Generation of homologous recombination deficiency (HRD) scores and mutational signatures}

Using the genome-wide CN data, a HRD score [24] was calculated for each tumour by summing the individual scores for Telomeric allelic imbalances (NtAI) [25], Large-scale 
State transitions (LSTs) [26] and Homologous recombination deficiency-Loss-ofheterozygosity (HRD-LOH) [27] following methods previously described [25-27].

Mutational signatures were defined by measuring the mutational context of somatic single nucleotide variation (SNVs) within the target regions. Somatic mutations located in the capture target region from tumour sequencing were analysed and enriched for likely somatic mutations using the following filters: called by two or more callers; alternate variant proportion more than 10\%; MAF $\leq 0.0001$ in ExAC, GnomAD, EVS, and 1000 Genomes in overall population and European population; variant recurrence is no more than 2 tumours, except for genes known to be frequently mutated genes in breast cancer (PIK3CA, TOX3, TP53, PTEN, SPEN, GATA3, KRAS and BRAF) [28]. For each of the 15 tumours with germline PALB2 mutation and 35 sporadic breast tumours (negative for germline BRCA1, BRCA2 and PALB2 LoF mutations), the somatic SNVs were categorized into six basic base substitutions $(C>A, C>G, C>T, T>A, T>C$ and $T>G)$ and subcategorized into 96 subcategories according to the trinucleotide context. To overcome the limitation of the small number of somatic mutations in each sample detected using the targeted sequencing panel (average 15 per sample), we pooled the mutations identified in each tumour set and analysed them as a single group. The mutational signatures were calculated and plotted using deconstructSigs [29] package in R v3.3.2 [30].

\section{Promoter methylation analysis of PALB2 and BRCA1}

DNA from tumour (200ng-500ng) and Female Genomic Reference DNA control (Promega, Wisconsin, USA) (500ng) were bisulfite treated using the EpiTect Fast DNA Bisulfite Kit 
(Qiagen, Valencia, CA, USA) according to the manufacturer's instructions. A 135bp region of the CpG island located in the PALB2 promoter, (chr16: $23,652,428$ to $23,652,562$; GRCh37/hg19) was PCR amplified as described by [31] using the primers 5'TTTTCGGTTTAGGGTTAATTGGGTT-3' (forward) 5' САССТTTTССТTСТССТСАСАACTAAA-3' (reverse). A 232bp region of the BRCA1 promoter region was amplified as described in [32] using the primers 5'GGTAGATTGGGTGGTTAATTTAGA-3' (forward) and CTAAAAAACCCCACAACCTATCC-3'. PCR products were then assessed for the extent of methylation by Sanger sequencing. 


\section{Results}

\section{PALB2 germline mutation families and tumour characteristics.}

Families with germline LoF mutations in PALB2 were identified by targeted gene panel sequencing of participants of the ViP study as reported previously [14]. Fifteen PALB2 LoF mutations carriers from 15 different families had archival FFPE breast cancer blocks available for analysis. Tumour and patient details are summarized in Table S1. Seven cases were ER-positive, including 4 that were also HER2 positive and 8 patients were "triple negative" (ER/PR/HER2 negative). Hormone receptor statuses were confirmed by CN data. All 15 cases were Grade 3 and invasive ductal cancers.

\section{Identification of somatic PALB2 mutations in tumours}

All exons and introns of PALB2 were sequenced to a mean read depth of 382 across the entire gene for all samples and $99 \%$ of the coding region bases were covered by $>20$ reads. The respective germline LoF PALB2 mutations were identified in all 15 cases and the variant allele read proportion was used as a measure of allelic status together with the allelic status of other germline SNPs detected across chromosome 16 and copy number data derived using off-target reads (Table 2; Figure S1). Four tumours harboured somatic point mutations in PALB2 (three truncating mutations and one missense mutation) with each retaining the germline LoF mutation (cases 0306571, 0279859, 0393382 and 0410313). The p.Gly1068Arg somatic missense mutation was predicted by in-silico tools Condel [33], PolyPhen2 [34] and SIFT [35] to be deleterious and the CADD score [36] was high at 15.7. In addition, the p.Gly1068Arg mutation is located within the functionally important WD40 domain of PALB2 and previously reported biochemical assays of PALB2 
harbouring WD40 domain missense variants p.Leu939Trp, p.Thr1030lle and p.Leu1143Pro have shown altered binding to RAD51C, RAD51 and BRCA2 [37]. A further 6 tumours showed strong evidence of $L O H$ across the PALB2 locus with all 6 retaining the PALB2 LoF allele (cases 0149217, 0241796, 0319619, 0388519, 0401044 and 0112255) as evidenced by the high LoF allele frequency (Table 2). The LoF allele frequency for case 0388519 was only 0.65 but this is consistent with the $\sim 30 \%$ tumour purity estimated for this case. Five tumours showed neither LOH nor somatic mutations at the locus (cases $0233453,0252381,0057225,0317466$ and 0324737 ). For case 0317466 , the variant allele frequency was 0.63 , but in the context of an estimated tumour purity of $80 \%$, this frequency is more consistent with allelic imbalance rather than $\mathrm{LOH}$. The presence of allelic imbalance is supported by SNP allele frequency data across the PALB2 locus and chromosome 16, which show variant allele frequencies clustering around 0.3 and 0.6 consistent with retention of the wild-type allele and duplication of the chromosome harbouring the mutant allele in this case. Overall, $67 \%(10 / 15)$ of the tumours from germline PALB2 LoF mutations carriers are concluded to be null for PALB2.

\section{Homologous recombination deficiency in PALB2 tumours}

We scored the extent of large-scale genomic alterations consistent with homologous recombination deficiency to assess for correlation with PALB2 status. Genome-wide copy number data were generated from off-target sequencing reads from the custom SureSelect targeted sequencing assay and a combined HRD score for genomic scar was generated by quantitating three separate parameters; NtAI, LST and HRD-LOH. In addition to the 15 PALB2 tumours, we also scored a cohort of 35 sporadic breast cancers 
that were assayed on the same SureSelect panel as the PALB2 tumours. Compared to sporadic breast cancers, the PALB2 tumours had significantly higher HRD scores regardless of PALB2 allelic status (Figure 1). Interestingly, there was no significant difference in the HRD scores between PALB2 heterozygotes and null tumours (Table 2; Figure 1) although one PALB2 heterozygous tumour (case 0324737) has a HRD score of 1 with a near-normal chromosome complement. None of the PALB2 tumours harboured additional germline or somatic mutations in hereditary breast cancer genes BRCA1, BRCA2, PTEN, ATM, ATR, BRIP1, RAD51 or any of the Fanconi anaemia genes that could explain the high HRD scores in the PALB2 heterozygous cancers, although somatic TP53 mutations were detected in $3 / 5$ of the PALB2 heterozygous tumours compared to 3/10 PALB2 null tumours (Table 2).

Mutation signatures were also assessed based on 110 SNVs identified in PALB2 germline mutation tumours (43 SNVs among the HET group and 67 among the NULL group) and 225 SNVs among the 35 sporadic breast tumours. The PALB2 tumours, but not the sporadic tumours, showed a prominent somatic mutation signature $3(36.5 \%$ versus $0 \%)$ consistent with defective homologous recombination [13,28,38] (Figure S2). Because of the small number of mutations it was not possible to derive reliable signatures for the PALB2 heterozygous and PALB2 null tumours separately. High HRD scores and somatic mutational signature 3 are both hallmark features of tumours with $B R C A 1$ or $B R C A 2$ mutations and HRD [26]. Methylation of the PALB2 and BRCA1 promoter regions was assessed for 4 of 5 PALB2 heterozygous and 2 of 10 PALB2 null tumours where sufficient quantity and quality of DNA was available. Neither the heterozygous nor null PALB2 
tumours showed any evidence of promoter hypermethylation in PALB2 or BRCA1 (Figure S3A, S3B).

The genomic loci commonly targeted by $\mathrm{CN}$ change were similar for the PALB2 tumours compared to the 35 sporadic cancers analysed with the same sequencing panel (Figure S4) with the exception of the presence of $\mathrm{CN}$ loss of chromosome $1 \mathrm{p}$ among the PALB2 tumours. Loss of $1 \mathrm{p}$ is not commonly reported in other large cohorts of sporadic breast cancer $(<6 \%$ of cases affected) [39]. The $1 p$ loss was more frequently seen among the PALB2 null tumours, although the number of cases is small.

\section{Discussion}

The genetic causes for a large proportion of hereditary breast cancer families remain unresolved (BRCAx families). Despite the implementation of targeted and whole exome sequencing strategies, efforts to identify new genes have been slow and hampered by the apparent high genetic heterogeneity of the majority of BRCAx families [40]. While many candidate genes have been proposed, the rarity of families carrying mutations means that many types of data will be required to corroborate a role for a particular gene in breast cancer predisposition. Based on archetypical familial cancer gene $R B 1$, the expectation for a recessive tumour suppressor gene is that tumours from individuals carrying germline pathogenic mutations will acquire a second somatic mutation in the wild-type allele, thus rendering the cell null for that gene. On this basis, sequencing of both germline and tumour DNA from individuals from BRCAx families is gaining favour in the search for new 
breast cancer genes, with the absence of a second hit disqualifying that gene from further investigating. However, while the "two hit" model clearly applies for genes such as BRCA1 $[41,42]$ and reviewed in [43], there have been doubts as to whether this is required for other hereditary breast cancer genes. It is certainly true that a second somatic genetic or epigenetic mutation may not be required if the germline mutation is acting as a dominant negative, such as for the ATM missense variant p. Val2424Gly and some TP53 missense mutations, but in these cases the tumour cell is effectively rendered null for each gene and are not genuine examples of a haplo-insufficient mode of cancer predisposition.

The role of PALB2 as a moderate to high penetrance breast cancer predisposition gene responsible for $\sim 1 \%$ to $2.4 \%$ of hereditary breast cancer families is now well established $[5,14]$. Based on limited analyses, PALB2 had emerged as an example of a hereditary breast cancer gene that may not require a second hit [9] with only $50 \%$ showing $\mathrm{LOH}$ of the wild-type allele (Table 1). While one study reported that $7 / 7$ germline PALB2 mutant tumours showed loss of the wild-type allele [11], the majority of the studies reported infrequent allelic loss. The strongest evidence to date of a bi-allelic inactivation requirement for PALB2 is from analysis of a single PALB2 tumour where exome sequencing identified a somatic nonsense mutation, presumably in the wild-type allele. This tumour also showed a very high LST score and a mutation signature 3 , both consistent with loss of homologous DNA repair capacity [13]. However, none of the other studies performed full exon sequencing of PALB2.

Our data suggest that the majority of PALB2 tumours do show bi-allelic inactivation and therefore conform to Knudsen's two hit model. In 6/6 cases where there was LOH at the 
PALB2 locus, all showed clear loss of the wild-type allele. While it is possible this was a chance occurrence, this seems unlikely in the context of the high rate of somatic point mutations identified in PALB2 (4 of 15 tumours, 27\%) compared to the somatic mutation rate of $0.9 \%$ reported in breast cancer in general (COSMIC database, Version 82 , released 3 August 2017 [44]). Consistent with this, the PALB2 null tumours all had high HRD scores (average 73.9) similar to scores reported for BRCA1 and BRCA2 breast tumours (average 63.1) [45]. PALB2 tumours also had significantly higher HRD scores than observed in our own cohort of sporadic breast cancers (average 26.2) and also those reported in the literature (average 28) [24]. Interestingly, with one exception, those tumours that retained the wild-type PALB2 allele had HRD scores similar to the PALB2 null tumours. None of the PALB2 heterozygous tumours showed evidence of PALB2 promoter hypermethylation, which is consistent with other studies showing hypermethylation at the PALB2 promoter is infrequent in breast cancer $[31,46]$. In addition, none of these tumours had detectable BRCA1 promoter methylation, or germline or somatic mutations in hereditary breast cancer genes that could explain such an HR defect, which raises the possibility that the functional copy of PALB2 in these cancers is abrogated via other epigenetic or posttranslational mechanisms.

Taken together, our findings show that $P A L B 2$ does generally conform to the two hit model and this is associated with a genomic scar consistent with loss of homologous DNA repair capacity. This has implications for the discovery of new breast cancer predisposition genes as it validates an emphasis on the presence of a "second hit" and a genomic scar signature consistent with loss of that gene. 


\section{Acknowledgements}

We thank Norah Grewal, Rebecca Driessen and the ViP study investigators at the Victorian and Tasmanian Familial Cancer Centres: Alison Trainer, Gillian Mitchell, Marion Harris, Geoff Lindeman, Thomas John, Yoland Antill, Ingrid Winship and Mary-Anne Young who provided germline and histological data. kConFab investigators, Heather Thorne, Lynda Williams and Genna Glavich for calling in the tumour blocks. David Byrne for scoring the tumour purity, and the investigators in the Peter MacCallum Cancer Centre Genomics Core Facility Gisela Mir Arnau and Tim Semple for sequencing our tumours, the Bioinformatics \& Cancer Genomics Laboratory for running our data through their bioinformatics pipelines. This work was supported by the National Breast Cancer Foundation, the Victorian Cancer Agency, and the National Health and Medical Research Council of Australia.

\section{Statement of author contributions}

IGC and PAJ conceived and designed the study. JEAL carried out experiments, acquired, analysed and interpreted the data. NL and SMR carried out experiments, acquired and analysed data. DC and KLG analysed and interpreted the data. SM and PAJ provided data and samples. $M Z$ ran our data through the bioinformatics pipeline. JEAL, IGC and PAJ were involved in drafting the manuscript and all authors read and provided critical feedback on the manuscript. 


\section{References}

1. Xia B, Sheng Q, Nakanishi K, et al. Control of BRCA2 cellular and clinical functions by a nuclear partner, PALB2. Mol Cell 2006; 22: 719-729.

2. Xia B, Dorsman JC, Ameziane N, et al. Fanconi anemia is associated with a defect in the BRCA2 partner PALB2. Nat Genet 2007; 39: 159-161.

3. Reid S, Schindler D, Hanenberg $\mathrm{H}$, et al. Biallelic mutations in PALB2 cause Fanconi anemia subtype FA-N and predispose to childhood cancer. Nat Genet 2007; 39: 162-164.

4. Rahman N, Seal S, Thompson D, et al. PALB2, which encodes a BRCA2interacting protein, is a breast cancer susceptibility gene. Nat Genet 2007; 39: 165167.

5. Antoniou AC, Casadei S, Heikkinen $\mathrm{T}$, et al. Breast-cancer risk in families with mutations in PALB2. N Engl J Med 2014; 371: 497-506.

6. Knudson AG, Jr. Mutation and cancer: statistical study of retinoblastoma. Proc Natl Acad Sci U S A 1971; 68: 820-823.

7. Tischkowitz M, Xia B, Sabbaghian N, et al. Analysis of PALB2/FANCN-associated breast cancer families. Proc Natl Acad Sci U S A 2007; 104: 6788-6793.

8. Balia C, Sensi E, Lombardi G, et al. PALB2: a novel inactivating mutation in a Italian breast cancer family. Fam Cancer 2010; 9: 531-536. 
9. Hartley $\mathrm{T}$, Cavallone $\mathrm{L}$, Sabbaghian $\mathrm{N}$, et al. Mutation analysis of PALB2 in BRCA1 and BRCA2-negative breast and/or ovarian cancer families from Eastern Ontario, Canada. Hered Cancer Clin Pract 2014; 12: 19.

10. Garcia MJ, Fernandez V, Osorio A, et al. Analysis of FANCB and FANCN/PALB2 fanconi anemia genes in BRCA1/2-negative Spanish breast cancer families. Breast Cancer Res Treat 2009; 113: 545-551.

11. Casadei S, Norquist BM, Walsh T, et al. Contribution of inherited mutations in the BRCA2-interacting protein PALB2 to familial breast cancer. Cancer Res 2011; 71: 2222-2229.

12. Pakkanen $\mathrm{S}$, Wahlfors $\mathrm{T}$, Siltanen $\mathrm{S}$, et al. PALB2 variants in hereditary and unselected Finnish prostate cancer cases. J Negat Results Biomed 2009; 8: 12.

13. Foo TK, Tischkowitz M, Simhadri S, et al. Compromised BRCA1-PALB2 interaction is associated with breast cancer risk. Oncogene 2017; 36: 4161-4170.

14. Thompson ER, Rowley SM, Li N, et al. Panel testing for familial breast cancer: Calibrating the tension between research and clinical care. J Clin Oncol 2016; 34: $1455-1459$.

15. Li H. Aligning sequence reads, clone sequences and assembly contigs with BWAMEM. 2013; arXiv:1303.3997v1 [q-bio.GN]. Available at https://arxiv.org/archive/qbio.

16. Van der Auwera GA, Carneiro MO, Hartl C, et al. From FastQ data to high confidence variant calls: the Genome Analysis Toolkit best practices pipeline. Curr Protoc Bioinformatics 2013; 43: 1110 11-33. 
17. Rimmer A, Phan H, Mathieson I, et al. Integrating mapping-, assembly- and haplotype-based approaches for calling variants in clinical sequencing applications. Nat Genet 2014; 46: 912-918.

18. Koboldt DC, Zhang Q, Larson DE, et al. VarScan 2: somatic mutation and copy number alteration discovery in cancer by exome sequencing. Genome Res 2012; 22: $568-576$.

19. McLaren W, Gil L, Hunt SE, et al. The Ensembl Variant Effect Predictor. Genome Biol 2016; 17: 122.

20. Lek M, Karczewski KJ, Minikel EV, et al. Analysis of protein-coding genetic variation in 60,706 humans. Nature 2016; 536: 285-291.

21. Exome Variant Server. http://evs.gs.washington.edu/EVS/. Last accessed September 2017.

22. Genomes Project C, Auton A, Brooks LD, et al. A global reference for human genetic variation. Nature 2015; 526: 68-74.

23. Kuilman $\mathrm{T}$, Velds $\mathrm{A}$, Kemper $\mathrm{K}$, et al. CopywriteR: DNA copy number detection from off-target sequence data. Genome Biol 2015; 16: 49.

24. Marquard AM, Eklund AC, Joshi T, et al. Pan-cancer analysis of genomic scar signatures associated with homologous recombination deficiency suggests novel indications for existing cancer drugs. Biomark Res 2015; 3: 9.

25. Birkbak NJ, Wang ZC, Kim JY, et al. Telomeric allelic imbalance indicates defective DNA repair and sensitivity to DNA-damaging agents. Cancer Discov 2012; 2: 366375. 
26. Popova T, Manie E, Rieunier G, et al. Ploidy and large-scale genomic instability consistently identify basal-like breast carcinomas with BRCA1/2 inactivation. Cancer Res 2012; 72: 5454-5462.

27. Abkevich V, Timms KM, Hennessy BT, et al. Patterns of genomic loss of heterozygosity predict homologous recombination repair defects in epithelial ovarian cancer. Br J Cancer 2012; 107: 1776-1782.

28. Nik-Zainal S, Davies H, Staaf J, et al. Landscape of somatic mutations in 560 breast cancer whole-genome sequences. Nature 2016; 534: 47-54.

29. Rosenthal R, McGranahan N, Herrero J, et al. DeconstructSigs: delineating mutational processes in single tumors distinguishes DNA repair deficiencies and patterns of carcinoma evolution. Genome Biol 2016; 17 : 31.

30. Team RDC. R: A Language and Environment for Statistical Computing. Vienna, Austria : the R Foundation for Statistical Computing 2011.

31. Mikeska T, Alsop K, Australian Ovarian Cancer Study G, et al. No evidence for PALB2 methylation in high-grade serous ovarian cancer. J Ovarian Res 2013; 6: 26.

32. Watanabe $\mathrm{Y}$, Maeda I, Oikawa $\mathrm{R}$, et al. Aberrant DNA methylation status of DNA repair genes in breast cancer treated with neoadjuvant chemotherapy. Genes Cells 2013; 18: 1120-1130.

33. Gonzalez-Perez A, Lopez-Bigas N. Improving the assessment of the outcome of nonsynonymous SNVs with a consensus deleteriousness score, Condel. Am J Hum Genet 2011; 88: 440-449. 
34. Adzhubei IA, Schmidt S, Peshkin L, et al. A method and server for predicting damaging missense mutations. Nat Methods 2010; 7: 248-249.

35. Ng PC, Henikoff S. Predicting deleterious amino acid substitutions. Genome Res 2001; 11: 863-874.

36. Kircher $\mathrm{M}$, Witten DM, Jain $\mathrm{P}$, et al. A general framework for estimating the relative pathogenicity of human genetic variants. Nat Genet 2014; 46: 310-315.

37. Park JY, Zhang F, Andreassen PR. PALB2: the hub of a network of tumor suppressors involved in DNA damage responses. Biochim Biophys Acta 2014; 1846: $263-275$.

38. Polak P, Kim J, Braunstein LZ, et al. A mutational signature reveals alterations underlying deficient homologous recombination repair in breast cancer. Nat Genet 2017; 49: 1476-1486.

39. Curtis C, Shah SP, Chin SF, et al. The genomic and transcriptomic architecture of 2,000 breast tumours reveals novel subgroups. Nature 2012; 486: 346-352.

40. Thompson ER, Gorringe KL, Rowley SM, et al. Prevalence of PALB2 mutations in Australian familial breast cancer cases and controls. Breast Cancer Res 2015; 17 : 111.

41. Esteller M, Silva JM, Dominguez G, et al. Promoter hypermethylation and BRCA1 inactivation in sporadic breast and ovarian tumors. J Natl Cancer Inst 2000; 92: 564-569.

42. Staff S, Isola J, Tanner M. Haplo-insufficiency of BRCA1 in sporadic breast cancer. Cancer Res 2003; 63: 4978-4983. 
43. Turner N, Tutt A, Ashworth A. Hallmarks of 'BRCAness' in sporadic cancers. Nat Rev Cancer 2004; 4: 814-819.

44. Simon A. Forbes, David Beare, Harry Boutselakis, et al. COSMIC: somatic cancer genetics at high-resolution. Nucleic Acids Res 2017; 45: D777-D783.

45. Telli ML, Timms KM, Reid J, et al. Homologous recombination deficiency (HRD) score predicts response to platinum-containing neoadjuvant chemotherapy in patients with triple-negative breast cancer. Clin Cancer Res 2016; 22: 3764-3773.

46. Potapova A, Hoffman AM, Godwin AK, et al. Promoter hypermethylation of the PALB2 susceptibility gene in inherited and sporadic breast and ovarian cancer. Cancer Res 2008; 68: 998-1002.

This article is protected by copyright. All rights reserved. 
Table 1. Summary of published tumour analyses of PALB2 carrier breast tumours

\begin{tabular}{|c|c|c|c|c|c|}
\hline \multirow{2}{*}{ Study } & \multirow{2}{*}{$\begin{array}{l}\text { No. of } \\
\text { Tumours } \\
\text { Studied }\end{array}$} & \multicolumn{2}{|c|}{ Allelic Status } & \multirow{2}{*}{$\begin{array}{c}\text { Somatic Point } \\
\text { Mutation }\end{array}$} & \multirow{2}{*}{ Technique } \\
\hline & & $\begin{array}{c}\text { LOH Wildtype } \\
\text { Allele }\end{array}$ & $\begin{array}{l}\text { LOH Mutant } \\
\text { Allele }\end{array}$ & & \\
\hline Tischkowitz (2007) [7] & 4 & 0 & 0 & Not tested & Site specific Sanger sequencing \\
\hline Garcia (2009) [10] & 1 & 1 & 0 & Not tested & Site specific Sanger sequencing \\
\hline Pakkanen (2009) [12] & 2 & 0 & 0 & Not tested & Site specific Sanger sequencing \\
\hline Balia (2010) [8] & 1 & 0 & 0 & Not tested & Site specific Sanger sequencing \\
\hline Casadei (2011) [11] & 7 & 7 & 0 & Not tested & Site specific Sanger sequencing \\
\hline Hartley (2014) [9] & 4 & 1 & 0 & Not tested & Site specific Sanger sequencing \\
\hline Foo (2017) [13] & 1 & 0 & 0 & 1 & Whole exome sequencing \\
\hline Total & 20 & 9 & 0 & 1 & \\
\hline
\end{tabular}

$\mathrm{LOH}$, loss of heterozygosity

This article is protected by copyright. All rights reserved. 
Table 2. Overall PALB2 carrier information and mutation summary of breast cancer tumours for this study

\begin{tabular}{|c|c|c|c|c|c|c|}
\hline Sample ID & $\begin{array}{l}\text { PALB2 } \\
\text { Status }\end{array}$ & PALB2 Mutation Nucleotide (Protein) & $\begin{array}{c}\text { Tp53 Mutation Nucleotide } \\
\text { (Protein) }\end{array}$ & $\begin{array}{l}\text { Tumour } \\
\text { Purity }\end{array}$ & HRD Score & Read \\
\hline 0233453 & HET & c.522_523delAA (p.Arg175ThrfsTer9) & c.1024C>T (p.Arg342Ter) & $60 \%$ & 73 & 85 \\
\hline 0252381 & HET & c.1037_1041delAAGAA (p.Lys346ThrfsTer13) & c.659A>G (p.Tyr220Cys) & $60 \%$ & 66 & 26 \\
\hline 0057225 & HET & c.3362delG (p.Gly1121ValfsTer3) & . & $45 \%$ & 83 & $2 \varepsilon$ \\
\hline 0317466 & HET & c.3113G>A (p.Trp1038Ter) & c.695T>A (p.lle232Asn) & $80 \%$ & 74 & 7 \\
\hline 0324737 & HET & c.3332delC (p.Pro1111LeufsTer13) & . & $65 \%$ & 1 & 70 \\
\hline 0149217 & NULL & 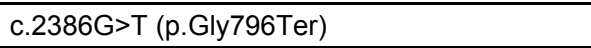 & . & $50 \%$ & 90 & 5 \\
\hline 0241796 & NULL & c.3113G>A (p.Trp1038Ter) & & $85 \%$ & 76 & 15 \\
\hline 0319619 & NULL & c.2915delT (p.Leu972ArgfsTer6) & . & $70 \%$ & 84 & 74 \\
\hline 0388519 & NULL & c.3113G>A (p.Trp1038Ter) & c.734G>T (p.Gly245Val) & $30 \%$ & 53 & 27 \\
\hline 0401044 & NULL & c.3113G>A (p.Trp1038Ter) & & $65 \%$ & 58 & 33 \\
\hline 0112255 & NULL & c.268G>T (p.Glu90Ter) & c.742C>T (p.Arg248Trp) & $75 \%$ & 101 & $2 \varepsilon$ \\
\hline \multirow{2}{*}{0306571} & \multirow{2}{*}{ NULL } & c.268G>T (p.Glu90Ter) & & \multirow{2}{*}{$60 \%$} & \multirow{2}{*}{75} & 27 \\
\hline & & ${ }^{*}$ c.3202G>C (p.Gly1068Arg) & & & & 26 \\
\hline \multirow{2}{*}{0279859} & \multirow{2}{*}{ NULL } & c.3113G >A (p.Trp1038Ter) & & \multirow{2}{*}{$80 \%$} & \multirow{2}{*}{61} & 4 \\
\hline & & "c.3193_3201+6delCTGAAATGGTAAGT (p.?) & & & & 4 \\
\hline \multirow{2}{*}{0393382} & \multirow{2}{*}{ NULL } & c.2771delC (p.Pro924GInfsTer11) & \multirow{2}{*}{ c.909_919+13del (p.?) } & \multirow{2}{*}{$60 \%$} & \multirow{2}{*}{91} & 5 \\
\hline & & ${ }^{*}$ c.876_898del (p.GIn292HisfsTer3) & & & & 3 \\
\hline \multirow{2}{*}{0410313} & \multirow{2}{*}{ NULL } & c.3507_3508delTC (p.His1170PhefsTer19) & & \multirow{2}{*}{$70 \%$} & \multirow{2}{*}{50} & \\
\hline & & ${ }^{*} \mathrm{c} .2630 \mathrm{G}>\mathrm{A}$ (p.Trp877Ter) & & & & \\
\hline
\end{tabular}

HET, Heterozygous tumour; NULL, tumour with bi-allelic inactivation; *indicates PALB2

somatic mutation; HRD score, sum of the individual NtAI, LSTs and HRD-LOH scores

This article is protected by copyright. All rights reserved. 
Figure Legend.

Figure 1. Large scale genomic alteration scores for 35 sporadic breast carcinomas, 10 PALB2 null and 5 PALB2 heterozygous tumours plotted as (A) HRD score, (B) NtAl, (C) LST and (D) HRD-LOH. $p$ values are shown for each group.

\section{SUPPLEMENTARY MATERIAL ONLINE}

\section{Supplementary materials and methods NO}

\section{Supplementary figure legends YES}

Table S1. Carrier subject details and tumour characteristics obtained from pathology reports

Figure S1. Analysis of Loss-of-heterozygosity and allelic imbalance across chromosome 16, including PALB2

Figure S2. Mutational signatures of $P A L B 2$ tumours versus sporadic breast tumours

Figure S3. Bisulfite sequencing of the PALB2 tumours within the PALB2 and BRCA1 promoter regions

Figure S4. Genome wide copy number plots of sporadic breast cancers vs PALB2 tumours 


\section{University Library}

\section{- M M N E R VA A gateway to Melbourne's research publications}

Minerva Access is the Institutional Repository of The University of Melbourne

\section{Author/s:}

Lee, JEA;Li, N;Rowley, SM;Cheasley, D;Zethoven, M;Mclnerny, S;Gorringe, KL;James,

$P A ; C a m p b e l l, ~ I G$

Title:

Molecular analysis of PALB2-associated breast cancers

Date:

2018-05-01

\section{Citation:}

Lee, J. E. A., Li, N., Rowley, S. M., Cheasley, D., Zethoven, M., Mclnerny, S., Gorringe, K. L., James, P. A. \& Campbell, I. G. (2018). Molecular analysis of PALB2-associated breast cancers. JOURNAL OF PATHOLOGY, 245 (1), pp.53-60. https://doi.org/10.1002/path.5055.

Persistent Link:

http://hdl.handle.net/11343/283590 